\title{
Sur un corps non dénombrable de nombres réels.
}

\author{
Rédigé d'après un mémoire posthume de \\ Michel Souslin (Moscou) \\ par \\ Casimir Kuratowski (Varsovie).
}

Le but de cette note est de définir un corps non dénombrable de nombres réels n'en contenant pas la totalité ${ }^{1}$ ).

1. Pour arriver à ce but nous allons construire un ensemble parfait $P_{1}$ et une suite infinie $P_{2}, P_{3}, \ldots P_{n}, \ldots$ telle que $P_{n}=P_{n-1}+$

1) Ce problème a été publié par M. Mazurkiewicz dans Fund. Math. I, 1920 (problème No 8). Un problème plus simple, mais tout à fait analogue était résolu par M. Łomnicki dans un art. Sur les fonetions multipériodiquès uniformes d'une variable réelle (C. R. de la Soc. des Sciences de Varsovio 1918). Il y était question de nommer un eusemble non dénombrable de nombres réels contenant toutes les différences $x-y$ de ses élérnents, mais distinct de l'ensemble de tous les nombres réels. En modifant légèrement la démonstration de $\mathrm{M}$. Eo mn icki on prouve qu'un exemple d'un tel ensemble est fourni par le plus petit ensemble qui contient tous les "nombres de Liouville" (c'est a dire: les nombres de la forme $\frac{\alpha_{1}}{10^{1 !}}+\frac{\alpha_{3}}{10^{3 !}}+\frac{\alpha_{3}}{10^{3}}+\ldots$ ) ainsi que toutrs les différences de ses éléments. Cet exemple peut servir aussi pour construire une fonction périodique ayant un ensemble non dénombrable de périodes; car - comme le provve M. K om nicki pour qu'un ensemble de nombres réels puisse être l'ensemble de périodes d'une fonction (de variable réelle) il faut et il suffit qu'il contienne toutes les différences do ses éléments. La fonction caractéristique d'un tel ensemble (c'est à dire, Ǵgale à 1 aux éléments de cet ensemble et égale à 0 aillears) admet cet ensemble comme l'ensemble de ses périodes. Ainsi, en particulier, Ia fonction caractéristique de l'ensemble $S$, détini dans cette note, a une infinité non dénombrable de périodes; cello de l'ensemble $E$ de la formule $[3$ \} en a un ensemble $n$ on mes a rable (au sens de Lebesgue). 
+l'ensemble de tous les nombres $x-y$ et $\frac{x}{y}$, où $x$ et $y$ appartiennent à $P_{n-1}$ et $|x| \geqslant \frac{1}{n}$ et $|y| \geqslant \frac{1}{n}$.

L'ensemble

$$
S=P_{1}+P_{2}+\ldots P_{n}+\ldots
$$

sera évidemment un corps non dénombrable. Pour qu'il ne contienne pas la totalité des nombres réels, il suffira de définir l'ensemble $P_{1}$ de telle façon que la mesure (au sens de Lebesgue) $m(S)$ de $S$ soit finie.

2. Soient $f(x, y)$ et $g(x, y)$ deux fonctions réelles continues pour toutes les valeurs non nulles des arguments 1). Etant donnés un nombre $\delta>0$ et deux intervalles (fermés) $I_{1}$ et $I_{2}$, nous désignerons par $f_{\delta}\left(I_{1}, I_{2}\right)$ l'ensemble des valeurs de la fonction $f(x, y)$ pour les arguments assujéttis aux conditions:

$$
|x| \geqslant \delta,|y| \geqslant \delta, x \text { appurtient à. } I_{\mathrm{r}} \text { et } y \text { à } I_{\mathrm{a}} \text {. }
$$

Soit $I$ la longueur de l'intervalle $I$. Lorsque $I_{1} \leqslant \delta$ et $I_{2} \leqslant \delta$ les $x$ et $y$ qui. satisfont aux conditions (1) forment deux intervalles; la fonction $f^{\prime}(x, y)$ y étant continue, $f_{\delta}\left(I_{1}, I_{2}\right)$ est un intervalle. Cet intervalle peut d'ailleurs etre vide ou se réduire à un seal point.

Les mêmes remarques concernent le symbole $g_{\delta}\left(I_{1}, I_{2}\right)$.

3 . Soit $\eta>\delta>0$. La fonction $f(x, y)$ étant unifformément continue pour les arguments appartenant aux intervalles $[-\eta,-\delta]$ et

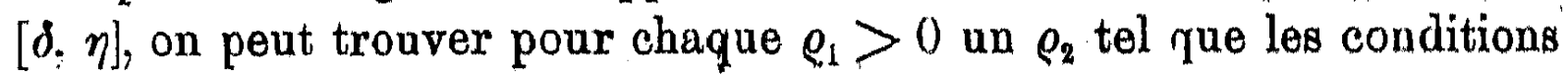

$$
I_{1}+I_{2}\left([-\eta,+\eta]^{2}\right), \quad \overline{I_{1}} \leqslant \varrho_{2} \text { et } \quad \tilde{I_{2}} \leqslant \varrho_{2}
$$

entraînent l'inégralité $\left.\overline{f_{\delta}\left(I_{1}\right.}, I_{2}\right) \leqslant \varrho_{1}$.

4. Nous allons construire l'ensemble parfait $P_{1}$ en définissant son système déterminant. Autrement dit, nous allons faire

y) On pourrait se borner ici au cas oi $f(x, y)=\frac{}{y}$ "Or, la nolution donnée par soualin - no dépondant pas do oes hypothesen apócialen - prete ì des généralisationa considérables.

2) Le symbole ${ }^{X} X Y^{\mu}$ veut diro quo l'onsemble $X$ est contenu dana $X$. 
correspondre à chaque système $\alpha_{1} \alpha_{2} \ldots \alpha_{n}$ composé de chiffres 0 et 1 un intervalle $D_{\alpha_{1} \alpha_{s} \ldots \alpha_{n}}$ de façon que

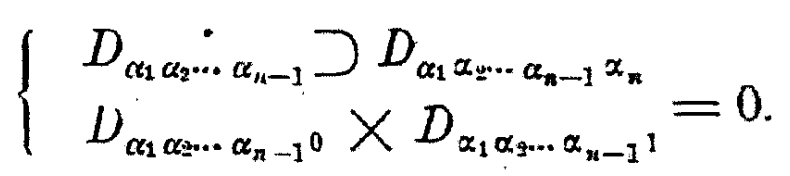

Un nombre $x$ sera dit élément de $P_{1}$, lorsqu'il existe une suite infinie $\alpha_{1}, \alpha_{2}, \ldots, \alpha_{n}, \ldots$ telle que chaque intervalle $D_{\alpha_{1} \alpha_{3} \ldots \alpha_{n}}$ contient $x$.

Soit $D$ l'intervalle $(0,1)$. D'après 3 , il existe dans $D$ deux intervalles disjoints $D_{0}$ et $D_{1}$ assujéttis à la condition suivante:

$$
I_{1}, I_{2}, \ldots I_{10}
$$

désignant la suite formée des intervalles $D_{0}, D_{1}$ et de tous les

$$
f_{1 / 2}\left(D_{i}, D_{i}\right) \text {. et } g_{1 / 2}\left(D_{i}, D_{j}\right) \text {. }
$$

oì $i, j=0$ ou $1,-$ on a

$$
\bar{I}_{1}+\bar{I}_{2}+\ldots \bar{I}_{10} \leqslant 1 \text {. }
$$

(Pour s'en convaincre on n'aurait qu'à poser dans le $\mathrm{N}^{0} 3: \eta=1$, $\delta=\frac{1}{2}, \varrho_{1}=\frac{1}{10}$ et $\varrho_{2} \leqslant \frac{1}{100}$. Les intervalles $D_{0}$ et $D_{1}$ peuvent être déterminés d'une façon effective, car leurs bornes peuvents toujours être supposées rationnelles.

Procédons par induction. Supposons que, pour un $n \geqslant 2$ donné, les intervalles disjoints $D_{\alpha_{1} \alpha_{n} \cdots \alpha_{n-1}}$ soient définis pour tous les systèmes $\alpha_{1}, \alpha_{2}, \ldots \alpha_{n-1}$ composés de 0 et 1 . En nous appayant sur 3 , nous pouvons définir une suite. $\left\{D_{\alpha_{1} \ldots a_{n}}\right\}$ d'intervalles satisfaisant à (2) et telle que la condition suivante soit réalisée:

Si $1^{0}: D_{1}^{0}, D_{3}^{0}, \ldots D_{k_{0}}^{0}$ désigne la suite des intervalles $\left\{D_{\alpha_{1} \alpha_{2} \ldots \alpha_{n}}\right\}$ (donc $k_{0}=2^{n}$ ),

$2^{0}:$ pour $1 \leqslant j \leqslant n, D_{1}^{j} . D_{2}^{j}, \ldots D_{k_{j}}^{j}$ désigne la suite de tous les intervalles

où

$$
f_{\frac{1}{n-\dagger^{-}}}\left(D_{s}^{t}, D_{t}^{r}\right) \text { et } g_{n+1}\left(D_{s}^{l}, D_{t}^{r}\right)
$$

$$
l \leqslant j-1, r \leqslant i-1, s \leqslant k_{i}, t \leqslant k_{r}
$$

$$
3^{0}: d_{n}=\sum_{\substack{j=0.1, \ldots, \ldots \\ i=1.2, \ldots k_{j}}} \bar{D}_{i}^{j}
$$

dans ces hypothèses on a $d_{x} \leqslant 1$ : 
Les intervalles $D_{\alpha_{1} \alpha_{2} \ldots \alpha_{n}}$ étant ainsi définis pour tous les $n$ naturels, l'ensemble parfait $P_{1}$ se trouve défini de même.

$$
\begin{aligned}
& \text { 5. Soit } f(x, y)=x-y \text { et } g(x, y)=\frac{x}{y} \text {. On roit aussitôt que } \\
& \qquad P_{1} \subset D, P_{i} \subset \sum_{i=1}^{10} I_{i}
\end{aligned}
$$

et qu'en général

$$
P_{n+1} \subset \sum_{\substack{j=0 \\ j=1,1, \ldots, n_{1}}} D_{i, 2, \ldots k_{j}}^{j}
$$

On a donc pour la mesure $m\left(P_{n+1}\right)$ l'inégalité

et, comme par définition

$$
m\left(P_{n+1}\right) \leqslant d_{n} \leqslant 1
$$

on en conclut que

$$
P_{1} \subset P_{2} \subset \ldots P_{n} \subset \ldots,
$$

$$
m(S)=\lim _{n \rightarrow \infty} d_{n} \leqslant 1 .
$$

L'ensemble $S$ répond donc au problème ${ }^{1}$ ).

Remarque sur la mesurabilité des corps numériques.

Dans un article Sur les distances des points des ensembles de mesure positive M. Steinbaus a prouvéé que, si $A$ ost un onsomble de nombres réels do mesure intérieure $\neq 0$ et $D$ est l'ensemble de toutes les différences $x-y$ oil $x$ et $y$ appartiennent $a A$, alors $D$ contiont un intervalle tout entier. On on conclat aussitôt que, si un corps de nombres réels ne contient pas la totalité de ces nombres, sa mesure intérieure est 0 . Le problème s'impose: existe-il un corps ne contenant pas la totalité des nombrẹs réels ct ayant la mesure extérieure $\neq 00^{2} ;$ ? ou; ce qui revient au même: existe-il un corps non mesurable de nombres réels?

Evidemment, ni l'ensemble $S$ de souslin (quí est une sommo d'uno suite

1) On pourrait démontrer que la construction de l'ensemble $S$ décrite tout à l'heure est réalisée, lorsqu'on pose, en particulier:

$$
\bar{D}_{\alpha_{1} \alpha_{2} \ldots \alpha_{n-1}}=n^{-n^{n}} \text {. }
$$

2) Fund. Math. I, p. 100, corollaire.

ग) La mesure intérieure ne peut être remplacéo dans l'énoncé de M. Stoinha u s par la mesure extérieure. Ceci résulte de l'existence d'un ensemble non mes a rable contenant toutes les différences de ses éléments. L'existence d'an tel ensemble a été démontréo par M. Lo minicki (l. c. p. 833) à l'aide de la „baso de $\mathrm{Hamel}$ a . Au même but pourrait servir d'ailleurs l'enemble $k$ de la formule (3) de cette note. 
d'ensembles fermés) ni les corps dénornbrables ne répondent à ce problème. L'existence d'un corps non mesurable, qui va être établie à présent, résulte de l'axiome du choix de $\mathrm{Z}$ ermelo1). Chaque ensemble de mesure intérieure $\neq 0$ contenant un ensemble parfait, il suffira pour notre but de prouver l'existence d'un corps qui ait des éléments communs avec chaque ensemble parfait et cependant ne contienne pas un nombre irrationnel $a$ donné d'avance (d'ailleurs, tont à fait arbitraire).

Désignons, pour un ensemble quelconque $A$ de nombres réels, par $C(A)$ le plus petit corps contenant $A$ et envisageons l'ensemble $R(A)$ de tous les nombres $x$ tels que $a$ appartient à $C(A+(x))-C(A)$. Ò voit anssitôt que $a$ peut ètre mis sous la forme $a=\frac{w(x)}{u(x)}$, où $w(x)$ et $u(x)$ sont deux polynomes à coéfficients appartenant à $C(A)$. Chaque $x$ est donc une racine d'une équation à coefficiente appartenant à $C(A+(\alpha)$. Par conséquent, si l'ensemble $A$ est intini, on a $\bar{A} \geqslant \overline{\bar{R}|\bar{A}|}$ ( $\bar{X}$ désignant, en général. la puissance de $X$ ).

Ceci établi, considérons la suite transfinie de tous les onsembles parfaits linéaires

$$
B_{0}, B_{1}, \ldots B_{\alpha}, \ldots \quad\left(\alpha<\Omega_{c}\right)
$$

$Q_{\mathfrak{C}}$ désignant le plus petit nombre ordinal de la paissance du contina.

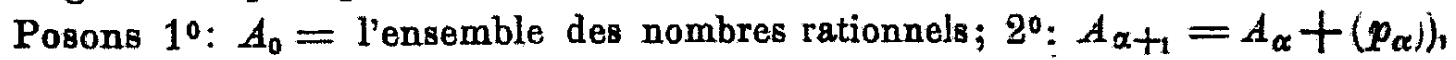
où $p_{\alpha}$ est un élément quelconque de $b_{\alpha}$ tel que $a$ n'appartient pas à $C\left(A_{\alpha}+\left(p_{\alpha}\right)\right.$; 30: lorsque $\alpha$ est un nombre limite, $A_{\alpha}=\sum_{\xi<\alpha} A_{\xi}$.

Les $p_{\alpha}$ existent pour $0<\alpha<\Omega_{C}$, car si $k_{\alpha}$ est l'ensemble de tous les $x$ tels que $a$ appartient à $\left.C\left(A_{\alpha}+(x)\right)-C_{(} A_{\alpha}\right)$, on a - comme nous l'arons dit -

$$
\overline{\bar{R}}_{\alpha} \leqslant \overline{\bar{A}}_{\alpha}<\overline{\bar{B}}_{\alpha}
$$

puisque $B_{\alpha}$ a la puissunce $c$ du continu. De plus $\overline{\overline{C\left(A_{\alpha}\right)}}<c$.

L'ensemble

$$
E=\sum_{\alpha<Q_{\mathfrak{c}}} C\left(A_{\alpha}\right)
$$

ost le corps dernandé, car il a des éléments communs avec chaque ensemble parfait $B_{\alpha}$ (à savoir: le point $p_{\alpha}$ ) et, en outre, il ne contient pas le nombre $a$. Sa mesure extérieure est donc $\infty$, tandis que sa mesure intérieure est 0 .

1) L'idée de cette démonstration est due à M. A. Zygmand. 